\title{
新型狮基膦金配合物的合成及其在炔烃水合反应中的催化作用研究
}

\author{
韩治军毛树兰彭晖皮云宵陈友 \\ 刘盛华* 余广鳌* \\ (农药与化学生物学教育部重点实验室 华中师范大学化学学院 武汉 430079)
}

\begin{abstract}
摘要 合成了四种新型䒢基膦金配合物，其结构经 ${ }^{1} \mathrm{H} \mathrm{NMR},{ }^{31} \mathrm{P}$ NMR 和元素分析表征，同时，两种配合物的结构通过 $\mathrm{X}$ 射线单晶衍射确证. 化合物 $\mathbf{2}$ 能高效地催化炔烃水合反应, 该催化反应具有反应条件温和、催化剂用量少、原子经济 性好、对环境友好等优点.
\end{abstract}

关键词 狮基; 膦; 金; 炔烃; 水合反应

\section{Synthetic and Structural Studies on Indenyl Phosphine Gold Complexes and Application as Catalyst for Hydration of Alkynes}

\author{
Han, Zhijun Mao, Shulan Peng, Hui Pi, Yunxiao $\quad$ Chen, You \\ Liu, Shenghua* Yu, Guangao* \\ (Key Laboratory of Pesticide \& Chemical Biology, Ministry of Education, College of Chemistry, \\ Central China Normal University, Wuhan 430079)
}

\begin{abstract}
Four novel indenyl phosphine gold complexes were prepared by the reaction of indenyl phosphine with AuCl$(\mathrm{SMe})_{2}$. All products $\mathbf{1} \sim \mathbf{4}$ have been fully characterized by elemental analysis, spectroscopy, and X-ray crystal diffraction techniques for $\mathbf{2}$ and $\mathbf{4}$. Complex $\mathbf{2}$ has been found efficiently to catalyze hydration of alkyne with the advantages of mild reaction conditions, less catalyst, excellent atomic economy and environmental friendly.
\end{abstract}

Keywords indenyl; phosphine; gold; alkyne; hydration reaction

羰基化合物在有机合成上具有特殊的地位，其在大 规模工业生产和精细化学品制备中都被广泛使用 ${ }^{[1]}$. 炔 烃的水合反应作为一个简便易行的合成羰基化合物的 方法，自从 1881 年被 Kucherov ${ }^{[2]}$ 首次发现以来就引起 了化学家们的广泛兴趣. 该反应以水和炔烃作为反应底 物, 具有原子经济性好以及环境友好等优点 ${ }^{[3]}$, 但是早 期使用的催化剂毒性较大, 因而化学家们一直专注于发 展毒性较低且更高效的催化剂. 目前已经使用过的金属 催化剂主要有铂 ${ }^{[4]}$ 、钉 ${ }^{[5]}$ 及其他金属有机化合物 ${ }^{[6]}$, 近年 来，使用金的配合物 ${ }^{[7,8]}$ 催化的炔烃水合反应已经占据 主导地位.

金的配合物催化的有机反应得到了广泛的研究，已 经能够使许多反应在温和的条件下进行, 且具有较高的
产率和化学选择性 ${ }^{[9]}$. 目前, 金的配合物催化的反应有 烯烃炔烃的环化反应 ${ }^{[10]}$ 、炔烃的氢化氨基化反应 ${ }^{[11]}$, 炔 烃的水合反应 ${ }^{[12]}$ 等，在国内也有一些关于金配合物催 化有机反应的研究, 如 Friedel-Crafts 烷基化反应 ${ }^{[13]}$, 烯 烃的胺基芳基化反应 ${ }^{[14]}$ 等. 本课题组此前合成了一系 列新型狮基膦配体，并用于钯催化 $\mathrm{C}-\mathrm{N}$ 偶联反应 ${ }^{[15,16]}$, 水相中的 $\mathrm{C}-\mathrm{C}$ 偶联反应 ${ }^{[17]}$ 以及钓催化的醇脱氢反应 ${ }^{[18]}$ 等. 本文合成了四种新型狮基膦金配合物. 化合物 2-(2,4,6-三甲基苯基)-1-狮基二环己基膦氯化金(2)可以 高效地催化炔烃水合反应，且该催化反应具有反应条件 温和、催化剂用量少、原子经济性好、对环境友好等优 点. 本研究对于发展新型高催化活性的金膦配合物具有 一定的意义.

\footnotetext{
*E-mail: chshliu@mail.ccnu.edu.cn; yuguang@mail.ccnu.edu.cn

Received January 23, 2014; revised February 22, 2014; published online March 12, 2014

Project supported by the National Natural Science Foundation of China (Nos. 21072071, 21272088).

国家自然科学基金(Nos. 21072071, 21272088)资助项目.
} 


\section{1 结果与讨论}

\section{1 狮基膦金配合物的合成与表征}

如 Scheme 1 中所示, 四种狮基膦配体(或其四氟硼 酸盐)在氮气保护下分别与 $\mathrm{AuCl}\left(\mathrm{SMe}_{2}\right)$ 反应, 得到四种 相应的狮基膦配体的金配合物 $\mathbf{1} \sim \mathbf{4}$, 这四种化合物在 溶液中会缓慢分解, 在固态时氮气保护下能稳定存在. 所得化合物都经过了 ${ }^{1} \mathrm{H} N M R 、{ }^{31} \mathrm{P} N \mathrm{~N}$ 、元素分析的 表征确认, 在 ${ }^{31} \mathrm{P} N M R$ 中, 化合物 $\mathbf{1} \sim \mathbf{4}$ 分别在 $\delta 34.28$, $28.72,46.34,30.82$ 处有一个单峰, 说明磷原子与金属中 心已经配位.

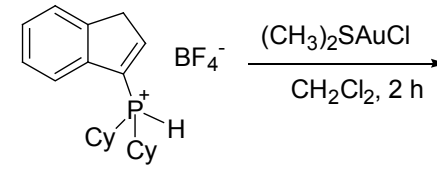<smiles>O=P(Cl)([Te]Cl)C1=CCc2ccccc21</smiles><smiles>Cc1cc(C)c(C2=C([P+](=O)[O-])c3ccccc3C2)c(C)c1</smiles>

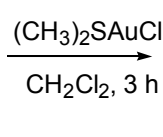<smiles>Cc1cc(C)c(C2=C(P(=O)(Cl)Cl)c3ccccc3C2)c(C)c1</smiles>

2

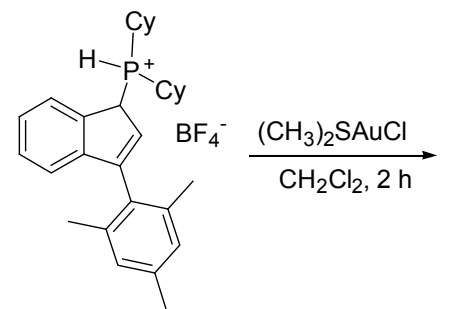

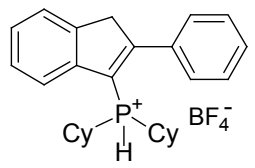
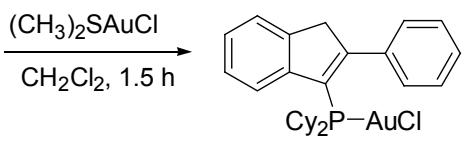

4

\section{Scheme 1}

\section{2 晶体结构}

化合物 2 的单晶结构如图 1 所示. 其晶体结构中 $\mathrm{C}(10)$ 与 $\mathrm{C}(13)$ 之间键长为 1.360(5) $\AA$, 属于碳碳双键. 2 , 4,6-三甲基苯基连在狮基的 2 位, $\mathrm{C}(4)$ 与 $\mathrm{C}(10)$ 之间键长 为 1.483(5) $\AA$, 磷原子与狮的 3 位碳相连, $\mathrm{C}(13)$ 与 $\mathrm{P}(1)$ 之间键长为 $1.812(3) \AA, \mathrm{Au}(1)$ 与 $\mathrm{P}(1)$ 之间键长为 2.2330(9) A. 键角 $\mathrm{P}(1)-\mathrm{Au}(1)-\mathrm{Cl}(1)$ 为 174.2(4) ${ }^{\circ}$, 接近 直线, 符合金配合物的配位方式. 化合物 4 的单晶结构 如图 2 所示, 其结构与化合物 2 非常相似, 其中, $\mathrm{C}(19)-\mathrm{C}(20)$ 键键长为 $1.354(16) \AA$, 比化合物 2 的 $\mathrm{C}(10)-\mathrm{C}(13)$ 键键长稍短, 也属于碳碳双键. 键角 $\mathrm{P}(1)-\mathrm{Au}(1)-\mathrm{Cl}(1)$ 为 $177.90(13)^{\circ}$, 接近直线. 化合物 2
和 4 晶体的部分键长和键角数据列于表 1 中.

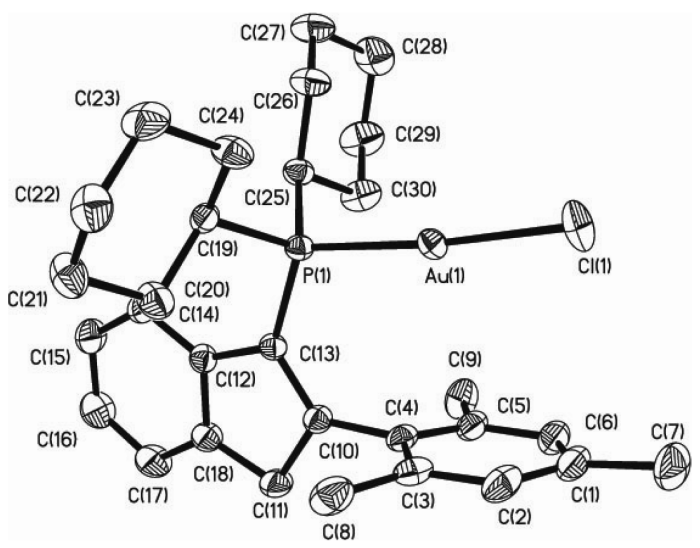

图 1 化合物 2 的单晶结构图

Figure 1 Molecular structure of compound 2

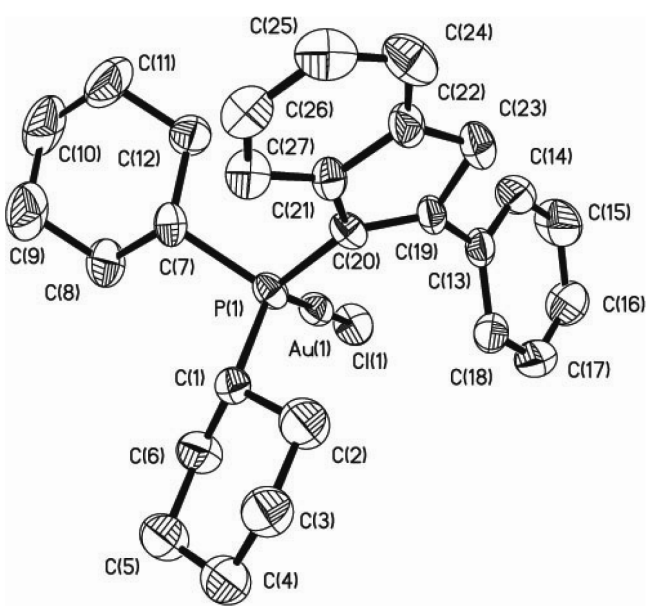

图 2 化合物 4 的单晶结构图

Figure 2 Molecular structure of compound 4

\section{3 化合物 2 催化的炔烃水合反应的条件优化}

在氮气保护下，化合物 2 (2 mol \%)为催化剂, 添加 $2 \mathrm{~mol}^{\circ} \mathrm{AgSbF}_{6}$, 控制温度 $80{ }^{\circ} \mathrm{C}$, 以 $1 \mathrm{mmol}$ 苯乙炔的 水合反应作为模板反应，对反应条件进行了优化. 实验 结果表明, 当水的体积为 $0.1 \mathrm{~mL}$, THF 的体积为 $1.0 \mathrm{~mL}$ 时，反应时间 $0.5 \mathrm{~h}$ 时产率只有 $22 \%$ (表 2, Entry 1), 而在 同样反应条件下，化合物 $\mathbf{1}, \mathbf{3}$ 和 4 对此反应没有催化作 用. 延长反应时间可以有效地提高反应的产率，当反应 时间为 $6 \mathrm{~h}$ 时，产率高达 94\%(表 2, Entries 2４). 增加 体系中水的量则会使反应的产率显著地下降(表 2, Entries $5 \sim 7)$, 当四氢呋喃和水的体积比降低为 $1: 1$ 时则 反应不会发生(表 2, Entries 5 9). 因此该反应的最优条 件为: 使用 $V(\mathrm{THF}): V\left(\mathrm{H}_{2} \mathrm{O}\right)=10: 1$ 的混合溶剂, 以化 合物 2 (2 mol\%) 为催化剂, $2 \mathrm{~mol} \% \mathrm{AgSbF}_{6}$ 为添加剂, $\mathrm{N}_{2}$ 保护下在 $80{ }^{\circ} \mathrm{C}$ 下反应 $6 \mathrm{~h}$. 
表 1 化合物 $\mathbf{2}$ 和 $\mathbf{4}$ 的部分键长 $(\AA)$ 与键角 $\left(^{\circ}\right)$

Table 1 Selected bond lengths $(\AA)$ and angles $\left({ }^{\circ}\right)$ for compound 2 and 4

\begin{tabular}{ll||ll}
\hline & 化合物 $\mathbf{2}$ & \multicolumn{1}{c}{ 化合物 $\mathbf{4}$} \\
\hline $\mathrm{Au}(1)-\mathrm{P}(1)$ & $2.2330(9)$ & $\mathrm{Au}(1)-\mathrm{P}(1)$ & $2.244(3)$ \\
$\mathrm{C}(13)-\mathrm{P}(1)$ & $1.812(3)$ & $\mathrm{Au}(1)-\mathrm{Cl}(1)$ & $2.315(4)$ \\
$\mathrm{C}(4)-\mathrm{C}(10)$ & $1.483(5)$ & $\mathrm{C}(7)-\mathrm{P}(1)$ & $1.857(13)$ \\
$\mathrm{Au}(1)-\mathrm{Cl}(1)$ & $2.2833(10)$ & $\mathrm{C}(19)-\mathrm{C}(20)$ & $1.354(16)$ \\
$\mathrm{C}(10)-\mathrm{C}(13)$ & $1.360(5)$ & $\mathrm{C}(19)-\mathrm{C}(23)$ & $1.527(17)$ \\
$\mathrm{C}(12)-\mathrm{C}(13)$ & $1.479(5)$ & $\mathrm{C}(20)-\mathrm{P}(1)$ & $1.832(12)$ \\
$\mathrm{P}(1)-\mathrm{Au}(1)-\mathrm{Cl}(1)$ & $174.2(4)$ & $\mathrm{P}(1)-\mathrm{Au}(1)-\mathrm{Cl}(1)$ & $177.90(13)$ \\
$\mathrm{C}(13)-\mathrm{P}(1)-\mathrm{Au}(1)$ & $114.58(11)$ & $\mathrm{C}(20)-\mathrm{P}(1)-\mathrm{Au}(1)$ & $113.8(4)$ \\
\hline
\end{tabular}

表 2 化合物 2 催化炔烃水合反应的条件优化 ${ }^{a}$

Table 2 Optimization of hydration of alkyne with catalyst 2

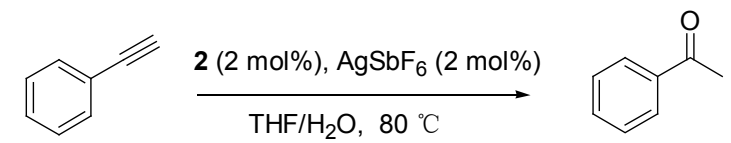

\begin{tabular}{cccc}
\hline Entry & $V(\mathrm{THF}): V\left(\mathrm{H}_{2} \mathrm{O}\right)$ & Time/h & Yield $^{b} / \%$ \\
\hline 1 & $10: 1$ & 0.5 & 22 \\
2 & $10: 1$ & 2.5 & 58 \\
3 & $10: 1$ & 5.0 & 73 \\
4 & $10: 1$ & 6.0 & 94 \\
5 & $5: 1$ & 6.0 & 48 \\
6 & $3: 1$ & 6.0 & 32 \\
7 & $2: 1$ & 6.0 & 7 \\
8 & $1: 1$ & 6.0 & - \\
9 & $1: 3$ & 6.0 & - \\
\hline
\end{tabular}

${ }^{a}$ Reaction conditions: $1.0 \mathrm{mmol}$ of alkyne, $0.02 \mathrm{mmol}$ of $2,0.02 \mathrm{mmol}$ of $\mathrm{AgSbF}_{6}, 80{ }^{\circ} \mathrm{C} .{ }^{b} \mathrm{GC}$ yield.

\section{4 化合物 2 催化的炔烃水合反应}

在最优反应条件下, 我们实现了一系列炔烃的水合 反应, 结果如表 3. 苯乙炔 $(\mathbf{5 a})$ 可转化为苯乙酮 $(\mathbf{6 a})$, 分 离产率高达 91\%(表 3, Entry 1), 对位甲氧基取代的苯乙 炔 $(5 \mathbf{b})$ 以 $72 \%$ 的产率转化为相应的酮 $6 \mathbf{b}$ (表 3, Entry 2), 而苯基丙炔 $(5 c)$ 可位置专一性的生成苯基乙基酮(6c), 产率达到 79\%, 具有一定空间位阻的二苯基乙炔 $(5 \mathrm{~d})$ 也 以 $79 \%$ 的产率生成相应的苠基苯基酮(6d), 具有大空间 位阻的 9-蒽基三甲基硅乙炔(5e) 能以 $61 \%$ 的产率转化为 9-葸基乙酮(6e).

\section{2 结论}

合成了四种新渘的茚基膦金配合物, 所得化合物都 通过 ${ }^{1} \mathrm{H}$ NMR, ${ }^{31} \mathrm{P} \mathrm{NMR}$, 元素分析进行表征, 同时, 利 用 $\mathrm{X}$ 射线单晶衍射确证了化合物 $\mathbf{2}$ 和 $\mathbf{4}$ 的分子结构. 化 合物 2 可催化多种炔烃的水合反应, 以中等到高的产率 得到相应的酮. 部分反应底物可位置专一性地得到产 物.
表 3 化合物 2 催化的炔烃水合反应 ${ }^{a}$

Table 3 Hydration of alkyne with catalyst 2

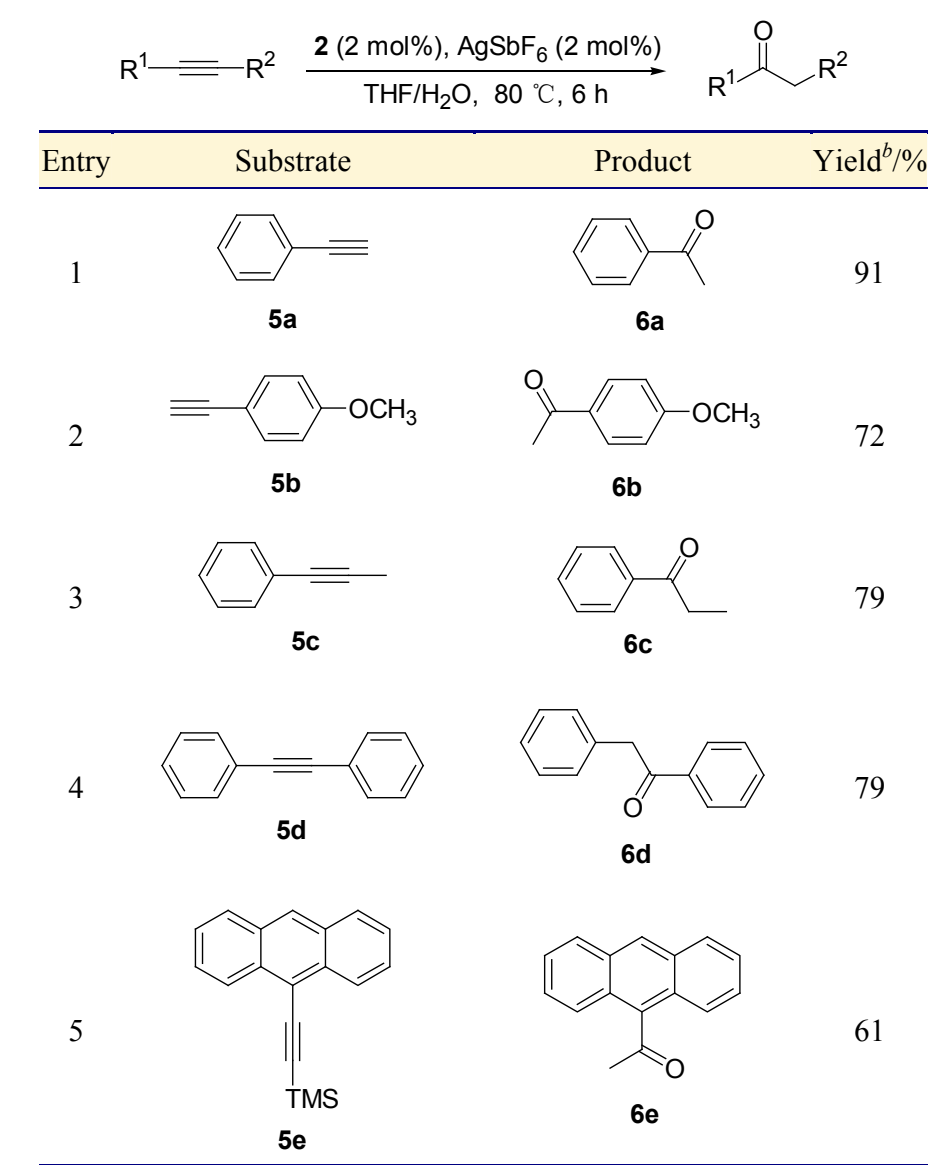

${ }^{a}$ Reaction conditions: $1.0 \mathrm{mmol}$ of alkyne, $1.0 \mathrm{~mL}$ of THF, $0.1 \mathrm{~mL}$ of $\mathrm{H}_{2} \mathrm{O}, 2$ mol\% 2, 2 mol\% $\mathrm{AgSbF}_{6}, 80{ }^{\circ} \mathrm{C} .{ }^{b}$ Isolated yield.

\section{3 实验部分}

\section{1 仪器和试剂}

气相色谱在 Agilent $6890 \mathrm{~N}$ 气相色谱仪上测定; ${ }^{1} \mathrm{H}$ NMR, ${ }^{31}$ P NMR 在 Varian Mercury Plus $400(400 \mathrm{MHz})$ 核 磁共振仪上测定 $\left({ }^{1} \mathrm{H}\right.$ NMR 以 TMS 作内标, ${ }^{31} \mathrm{P}$ NMR 以 $85 \%$ 的 $\mathrm{H}_{3} \mathrm{PO}_{4}$ 为外标); 元素分析在 Elementar Vario EL 型分析仪上测定.二氯甲烷经过 $\mathrm{CaH}_{2}$ 除水后使用. $\mathrm{AuCl}\left(\mathrm{SMe}_{2}\right)^{[19]} ， 2-(2,4,6-$ 三甲基苯基)-1-狮基二环己基 
膦 ${ }^{[16]}, 2$-苯基-1-苑基二环已基膦四氟嗍酸盐 ${ }^{[15]}$ 参照文献 中的方法合成. 金属有机化合物的合成采用无水无氧操 作技术. 其它试剂均为市售分析纯.

\section{2 实验方法}

1-狮基二环己基膦氯化金(1)的合成: 氮气保护下, 将 1-狮基二环己基膦四氟硼酸盐 $(103.0 \mathrm{mg}, 0.26 \mathrm{mmol}$ ), $\mathrm{AuCl}\left(\mathrm{SMe}_{2}\right)(97.0 \mathrm{mg}, 0.33 \mathrm{mmol})$ 加入到 Schlenk 管中, 然后加入 $5 \mathrm{~mL}$ 的 $\mathrm{CH}_{2} \mathrm{Cl}_{2}$, 室温下反应 $2 \mathrm{~h}$. 反应完毕后, 减压脱去溶剂, 粗产品用硅胶色谱柱分离, $\mathrm{CH}_{2} \mathrm{Cl}_{2}$ 洗脱, 得到白色固体 $(96.0 \mathrm{mg})$, 产率为 $68 \%$.

2-(2,4,6-三甲基苯基)-1-狮基二环己基膦氯化金 (2) 的合成: 氮气保护下, 将 2-(2,4,6-三甲基苯基)-1-狮基二 环已基膦(129.0 mg, $0.25 \mathrm{mmol}), \mathrm{AuCl}\left(\mathrm{SMe}_{2}\right)(88.4 \mathrm{mg}$, $0.30 \mathrm{mmol}$ )加入到 Schlenk 管中, 然后加入 $5 \mathrm{~mL}$ 的 $\mathrm{CH}_{2} \mathrm{Cl}_{2}$, 室温下反应 $3 \mathrm{~h}$, 反应完毕后, 减压脱去溶剂, 粗产品用硅胶色谱柱分离, $\mathrm{CH}_{2} \mathrm{Cl}_{2}$ 洗脱, 得到白色固体 (143.0 mg), 产率 $86 \%$.

1-(2,4,6-三甲基)苯基-3-狮基二环己基膦氯化金(3) 的合成: 氮气保护下, 将 1-(2,4,6-三甲基)苯基-3-狮基二 环已基膦四氟硼酸盐(129.0 mg, 0.25mmol), $\mathrm{AuCl}\left(\mathrm{SMe}_{2}\right)$ $(88.0 \mathrm{mg}, 0.30 \mathrm{mmol})$ 加入到 Schlenk 管中, 然后加入 5 $\mathrm{mL} \mathrm{CH} \mathrm{Cl}_{2}$, 室温下反应 $2 \mathrm{~h}$, 反应完毕后, 减压脱去溶 剂, 粗产品用硅胶柱分离, $\mathrm{CH}_{2} \mathrm{Cl}_{2}$ 洗脱, 得白色固体 $(85.0 \mathrm{mg})$, 产率 $51 \%$.

2-苯基-1-狮基二环已基膦氯化金(4)的合成：氮气 保护下, 将 2-苯基-1-狮基二环已基膦四氟硼酸盐(143.0 $\mathrm{mg}, 0.30 \mathrm{mmol}), \mathrm{AuCl}\left(\mathrm{SMe}_{2}\right)(106.0 \mathrm{mg}, 0.36 \mathrm{mmol})$ 加入

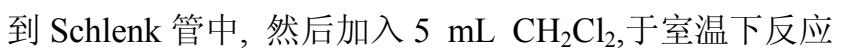
$1.5 \mathrm{~h}$, 反应完毕后, 减压脱去溶剂, 粗产品用硅胶柱分 离, $\mathrm{CH}_{2} \mathrm{Cl}_{2}$ 洗脱, 得白色固体 $(74.0 \mathrm{mg}$ ), 产率 $40 \%$.

化合物 2 催化的炔烃水合反应条件优化: 在氮气保 护下，向 $15 \mathrm{~mL}$ 耐压管中加入化合物 2 (13.3 mg, 0.02 $\mathrm{mmol}), \mathrm{AgSbF}_{6}(6.8 \mathrm{mg}, 0.02 \mathrm{mmol})$, 炔烃 $(1.00 \mathrm{mmol})$, $\mathrm{H}_{2} \mathrm{O}$, THF, 加入十六烷作为内标, 反应温度和反应时间 按照表 2 列出的条件进行. 反应完毕后, 冷却至室温, 二氯甲烷 $/ \mathrm{H}_{2} \mathrm{O}$ 萃取, flash 柱层析分离(硅胶: $200 \sim 300$ 目; 二氯甲烷作为洗脱剂)得到混合物, 在气相色谱仪 上测定.

化合物 2 催化的炔烃水合反应: 在氮气保护下, 向 $15 \mathrm{~mL}$ 耐压管中加入化合物 $2(13.3 \mathrm{mg}, 0.02 \mathrm{mmol})$ 、 $\mathrm{AgSbF}_{6}(6.8 \mathrm{mg}, 0.02 \mathrm{mmol})$ 、炔烃 $(1.00 \mathrm{mmol}) 、 \mathrm{H}_{2} \mathrm{O}(0.1$ $\mathrm{mL}), \mathrm{THF}(1.0 \mathrm{~mL}), 80{ }^{\circ} \mathrm{C}$ 反应 $6 \mathrm{~h}$. 反应完毕后, 冷却至 室温, 二氯甲烷 $/ \mathrm{H}_{2} \mathrm{O}$ 萃取, 柱层析分离(硅胶: $200 \sim 300$ 目; 石油醚或正己烷与二氯甲烷的混合洗脱剂)得到产 物.

\section{3 化合物数据}

1-狮基二环己基膦氯化金(1): ${ }^{1} \mathrm{H}$ NMR $(400 \mathrm{MHz}$, $\left.\mathrm{CDCl}_{3}\right) \delta: 7.83(\mathrm{~d}, J=7.4 \mathrm{~Hz}, 1 \mathrm{H}), 7.54(\mathrm{~d}, J=7.4 \mathrm{~Hz}$, 1H), $7.27 \sim 7.39$ (m, 3H), 3.6 (s, 2H), 2.36 (br, $2 \mathrm{H}), 2.11$ (br, 2H), $1.86(\mathrm{br}, 2 \mathrm{H}), 1.16 \sim 1.74(\mathrm{~m}, 16 \mathrm{H}) ;{ }^{31} \mathrm{P}$ NMR $\left(243 \mathrm{MHz}, \mathrm{CDCl}_{3}\right) \delta: 34.28$. Anal. calcd for $\mathrm{C}_{21} \mathrm{H}_{29} \mathrm{AuClP}$ : C 46.29, H 5.36; found C 46.31, H 5.33.

2-(2,4,6-三甲基苯基)-1-狮基二环己基膦氯化金(2): ${ }^{1} \mathrm{H}$ NMR $\left(400 \mathrm{MHz}, \mathrm{CDCl}_{3}\right) \delta: 7.55(\mathrm{~d}, J=7.6 \mathrm{~Hz}, 2 \mathrm{H})$, $7.26 \sim 7.39(\mathrm{~m}, 2 \mathrm{H}), 7.00(\mathrm{~s}, 2 \mathrm{H}), 3.70(\mathrm{~s}, 2 \mathrm{H}), 2.50(\mathrm{br}$, 2H), 2.42 (s, 3H), 2.09 (s, 6H), $1.18 \sim 2.09(\mathrm{~m}, 20 \mathrm{H}) ;{ }^{31} \mathrm{P}$ NMR $\left(243 \mathrm{MHz}, \mathrm{CDCl}_{3}\right) \delta$ : 28.72. Anal. calcd for $\mathrm{C}_{30} \mathrm{H}_{39} \mathrm{AuClP}$ : C 54.35, H 5.93; found C 54.32, H 5.97.

1-(2,4,6-三甲基)苯基-3-狮基二环己基膦氯化金(3): ${ }^{1} \mathrm{H}$ NMR (400 MHz, $\mathrm{CDCl}_{3}$ ) $\delta: 8.09$ (br, 1H), 7.30 7.33 (m, 2H), $6.96 \sim 7.01(\mathrm{~m}, 3 \mathrm{H}), 6.37$ (s, 1H), 4.43 (d, $J=16$ $\mathrm{Hz}, 1 \mathrm{H}), 2.35$ (s, 3H), 2.16 (s, 3H), 2.02 (s, 3H), 1.14 $2.08(\mathrm{~m}, 22 \mathrm{H}) ;{ }^{31} \mathrm{P}$ NMR $\left(243 \mathrm{MHz}, \mathrm{CDCl}_{3}\right) \delta: 46.34$. Anal. calcd for $\mathrm{C}_{30} \mathrm{H}_{39} \mathrm{AuClP}$ : C 54.35, H 5.93; found C 54.37, H 5.91.

2-苯基-1-狮基二环已基膦氯化金(4): ${ }^{1} \mathrm{H}$ NMR (400 $\left.\mathrm{MHz}, \mathrm{CDCl}_{3}\right) \delta: 7.66(\mathrm{~d}, J=8.4 \mathrm{~Hz}, 1 \mathrm{H}), 7.21 \sim 7.54(\mathrm{~m}$, $8 \mathrm{H}), 3.86$ (s, 2H), 2.48 (br, 2H), 2.07 (br, 2H), 1.84 (br, $2 \mathrm{H}), 1.20 \sim 1.72(\mathrm{~m}, 16 \mathrm{H}) ;{ }^{31} \mathrm{P}$ NMR $\left(243 \mathrm{MHz}, \mathrm{CDCl}_{3}\right) \delta$ : 30.82. Anal. calcd for $\mathrm{C}_{27} \mathrm{H}_{33} \mathrm{AuClP}$ : C 52.23, H 5.36; found C 52.26, H 5.32 .

苯乙酮(6a): ${ }^{1} \mathrm{H}$ NMR $\left(400 \mathrm{MHz}, \mathrm{CDCl}_{3}\right) \delta: 7.96(\mathrm{~d}$, $J=8.0 \mathrm{~Hz}, 2 \mathrm{H}), 7.44 \sim 7.56(\mathrm{~m}, 3 \mathrm{H}), 2.61(\mathrm{~s}, 3 \mathrm{H})$. 氢谱数 据与文献值一致 ${ }^{[20]}$.

对甲氧基苯乙酮(6b): ${ }^{1} \mathrm{H}$ NMR (400 $\mathrm{MHz}, \mathrm{CDCl}_{3}$ ) $\delta: 7.93(\mathrm{~d}, J=8.0 \mathrm{~Hz}, 2 \mathrm{H}), 6.92(\mathrm{~d}, J=8.0 \mathrm{~Hz}, 2 \mathrm{H}), 3.85$ $(\mathrm{s}, 3 \mathrm{H}), 2.54(\mathrm{~s}, 3 \mathrm{H})$. 氢谱数据与文献值一致 ${ }^{[21]}$.

苯基乙基酮(6c): ${ }^{1} \mathrm{H}$ NMR $\left(400 \mathrm{MHz}, \mathrm{CDCl}_{3}\right) \delta: 7.88$ (br, 2H), $7.36 \sim 7.48(\mathrm{~m}, 3 \mathrm{H}), 7.36(\mathrm{~s}, 2 \mathrm{H}), 2.92$ (t, $J=8.0$ $\mathrm{Hz}, 2 \mathrm{H}), 1.15(\mathrm{~d}, J=8.0 \mathrm{~Hz}, 3 \mathrm{H})$. 氢谱数据与文献值一 致 ${ }^{[21]}$.

苯基苄基酮(6d): ${ }^{1} \mathrm{H}$ NMR (400 $\left.\mathrm{MHz}, \mathrm{CDCl}_{3}\right) \delta$ : $8.00 \sim 8.03(\mathrm{~m}, 2 \mathrm{H}), 7.54 \sim 7.56(\mathrm{~m}, 1 \mathrm{H}), 7.46(\mathrm{~d}, J=7.8$ $\mathrm{Hz}, 2 \mathrm{H}), 7.33(\mathrm{~d}, J=7.5 \mathrm{~Hz}, 2 \mathrm{H}), 7.25 \sim 7.28(\mathrm{~m}, 3 \mathrm{H})$, $4.29(\mathrm{~s}, 2 \mathrm{H})$. 氢谱数据与文献值一致 ${ }^{[7 \mathrm{c}]}$.

9-葸基甲基酮(6e): ${ }^{1} \mathrm{H}$ NMR (400 $\mathrm{MHz}, \mathrm{CDCl}_{3}$ ) $\delta$ : 8.47 (s, 1H), 8.01 (d, $J=8.0 \mathrm{~Hz}, 2 \mathrm{H}), 7.84$ (d, $J=8.0 \mathrm{~Hz}$, $2 \mathrm{H}), 7.48 \sim 7.52(\mathrm{~m}, 4 \mathrm{H}), 2.81(\mathrm{~s}, 3 \mathrm{H})$. 氢谱数据与文献 值一致 ${ }^{[22]}$. 


\section{4 晶体结构测定}

挑选适当的晶体, 在 Bruker SMART CCD 单晶衍 射仪上，采用石墨单色化的 $\mathrm{MoK} \alpha$ 射线 $(\lambda=0.71073 \AA)$ 收集数据. 全部数据均经过 SADABS 吸收校正, 晶体结 构采用 SHELXS-97 程序包解析, 对全部非氢原子及其 各向异性热参数进行全矩阵最小二乘法修正. 配合物的 晶体学数据见辅助材料, 数据存于英国剑桥数据中心, CCDC 号分别为: 2, CCDC-981565; 4, CCDC-981566.

辅助材料(Supporting Information) 狮基膦金配合物 的 ${ }^{1} \mathrm{H}$ NMR, ${ }^{31} \mathrm{P}$ NMR 和元素分析报告, 化合物 $\mathbf{2}$ 和 $\mathbf{4}$ 的 部分晶体数据以及炔烃水合反应产物的 ${ }^{1} \mathrm{H}$ NMR. 这些 材料可以免费从本刊网站(http://sioc-journal.cn/)上下载.

\section{References}

[1] Otera, J. Modern Carbonyl Chemistry, Wiley-VCH, Weinheim, Germany, 2000.

[2] Kucherov, M. Chem. Ber. 1881, 14, 1540.

[3] Trost, B. M. Science 1991, 254, 1471.

[4] (a) Hiscox, W.; Jennings, P. W. Organometallics 1990, 9, 1997. (b) Francisco, L. W.; Moreno, D. A.; Atwood, J. D. Organometallics 2001, 20, 4237.

[5] (a) Tokunaga, M.; Wakatsuki, Y. Angew. Chem., Int. Ed. 1998, 37, 2867.

(b) Suzuki, T.; Tokunaga, M.; Wakatsuki, Y. Org. Lett. 2001, 3, 735 .

[6] (a) Janout, V.; Regen, S. L. J. Org. Chem. 1982, 47, 3331.

(b) Tachinami, T.; Nishimura, T.; Ushimaru, R.; Noyori, R.; Naka, H. J. Am. Chem. Soc. 2013, 135, 50.

[7] (a) Fukuda, Y.; Utimoto, K. J. Org. Chem. 1991, 56, 3729.

(b) Marion, N.; Ramón, R. S.; Nolan, S. P. J. Am. Chem. Soc. 2009, 131,448 .

(c) Wang, D.; Cai, R.; Sharma, S.; Jirak, J.; Thummanapelli, S. K.;
Akhmedov, N. G.; Zhang, H.; Liu, X.; Petersen, J. L.; Shi, X. J. Am. Chem. Soc. 2012, 134, 9012.

[8] (a) Nun, P.; Ramón, R. S.; Gaillard, S.; Nolan, S. P. J. Organomet. Chem. 2011, 696, 7 .

(b) Leyva, A.; Corma, A. J. Org. Chem. 2009, 74, 2067.

[9] Li, Z.; Brouwer, C.; He, C. Chem. Rev. 2008, 108, 3239.

[10] Luzung, M. R.; Markham, J. P.; Toste, F. D. J. Am. Chem. Soc. 2004, 126, 10858.

[11] Mizushima, E.; Hayashi, T.; Tanaka, M. Org. Lett. 2003, 5, 3349.

[12] Almóssy, A.; Nagy, C. E.; Bényei, A. C.; Joó, F. Organometallics 2010, 29, 2484.

[13] (a) Wang, X.; Lai, Y.; Wu, H.; Zhang, J.; Li, Y. Chin. J. Org. Chem. 2009, 29, 432 (in Chinese).

(王翔, 赖媛媛, 吴宏, 张建明, 李永建, 有机化学, 2009, 29, 432.)

(b) Zhang, Y; Zhu, C. Chin. J. Org. Chem. 2012, 32, 2283. (in Chinese).

(张艳, 朱成建, 有机化学, 2012, 32, 2283.)

[14] Zhang, R.; Xu, Q.; Shi, M. Acta Chim. Sinica 2012, 70, 1593 (in Chinese). (张睿, 徐琴, 施敏, 化学学报, 2012, 70, 1593.)

[15] Chen, L.; Yu, G. A.; Li, F.; Zhu, X.; Zhang, B.; Guo, R.; Li, X.; Yang, Q.; Jin, S.; Li, C.; Liu, S. H. J. Organomet. Chem. 2010, 695, 1768.

[16] Hao, X.; Yuan, J.; Yu, G.-A.; Qiu, M.-Q.; She, N.-F.; Sun, Y.; Zhao, C.; Mao, S.-L.; Yin, J.; Liu, S.-H. J. Organomet. Chem. 2012, 706, 99.

[17] Mao, S.-L.; Sun, Y.; Yu, G.-A.; Zhao, C.; Han, Z.-J.; Yuan, J.; Zhu, X.; Yang, Q.; Liu, S. -H. Org. Biomol. Chem. 2012, 10, 9410.

[18] Yuan, J.; Sun, Y.; Yu, G.-A.; Zhao, C.; She, N.-F.; Mao, S.-L.; Huang, P.-S.; Han, Z.-J.; Yin, J.; Liu, S. H. Dalton Trans. 2012, 41, 10309.

[19] Hooper, T. N.; Butts, C. P.; Green, M.; Haddow, M. F.; McGrady, J. E.; Russell, C. A. Chem. Eur. J. 2009, 15, 12196.

[20] Goossen, L. J.; Manjolinho, F.; Khan, B. A.; Rodríguez, N. J. Org. Chem. 2009, 74, 2620.

[21] Chen, Z.-W.; Ye, D.-N.; Qian, Y.-P.; Ye, M.; Liu, L.-X. Tetrahedron 2013, 69, 6116.

[22] Gatti, N. Tetrahedron Lett. 1990, 31, 3933. 